\title{
Emociones frente a la pandemia del COVID-19 de los alumnos del Área Económico Administrativa de la Universidad Tecnológica de León
}

\section{Emotions in the face of the COVID-19 pandemic of the students of the Administrative Economic Area of the Technological University of León}

\author{
MARQUEZ-DE ANDA, Camilo†*, QUEZADA-FLORES, Ma. De la Luz, SERRANO-TORRES, Ma. \\ Guadalupe y MEJIA-GUERRERO, Mauricio
}

Universidad Tecnológica de León

ID $1^{\mathrm{er}}$ Autor: Camilo, Marquez-De Anda / ORC ID: 0000-0002-6800-0811

ID 1 ${ }^{\mathrm{er}}$ Coautor: Ma. De la Luz, Quezada-Flores / ORC ID: 0000-0002-4726-2695, CVU CONACYT ID: 801109

ID $2^{\text {do }}$ Coautor: Ma. Guadalupe, Serrano-Torres / ORC ID: 0000-0003-2229-6925, CVU CONACYT ID: 679788

ID $3^{\text {er }}$ Coautor: Mauricio Mejía-Guerrero / ORC ID: 0000-0002-6087-0150

DOI: $10.35429 / J T M S .2020 .19 .6 .1 .11$

Recibido 04 de Septiembre 2020; Aceptado 30 Diciembre, 2020

\begin{abstract}
Resumen
La presente investigación se refiere a la emocionalidad que existe en los estudiantes de la Universidad Tecnológica de León. El objetivo es analizar la situación que están viviendo los estudiantes de esta universidad, para estar en posibilidades de diseñar un plan pertinente para su apoyo eficiente, que permita el desarrollo profesional de este sector de la población; mediante una investigación sobre sus emociones y sentimientos causado por la pandemia del COVID 19 y por el estado de reclusión al que se vieron obligados. Este capítulo forma parte de una investigación extensa, en cuanto a las variables investigadas y el tamaño de la muestra. Del instrumento de recolección de información que se aplicó, se tomaron los resultados de los efectos de estos factores, en cuanto a las emociones y sentimientos y solamente de los alumnos de la Universidad Tecnológica de León. En los resultados encontramos, que están conscientes de que la posibilidad de contagio existe. El confinamiento en sus hogares, les proporciona seguridad y tranquilidad, sin embargo, también les causa ansiedad, frustración, preocupación y temor, por la situación que viven a causa de esta pandemia. A pesar de lo anteriormente expuesto, son optimistas sobre el futuro, piensan que las condiciones van a mejorar.
\end{abstract}

COVID-19, Emocionalidad, Pandemia

\begin{abstract}
This research refers to the emotionality that exists in students of the Universidad Tecnológica de León. The objective is to analyze the situation that the students of this university are experiencing, to be able to design a plan relevant to their efficient support, which allows the professional development of this sector of the population; through research into their emotions and feelings caused by the COVID 19 pandemic and the state of detention to which they were forced. This chapter is part of extensive research into the variables investigated and the sample size. From the information collection instrument that was applied, the results of the effects of these factors were taken, in terms of emotions and feelings and only of students of the Technological University of León. In the results we find that they are aware that the possibility of contagion exists. Confinement in their homes provides them with security and tranquility, however, it also causes them anxiety, frustration, concern and fear, because of the situation they live because of this pandemic. However, they are optimistic about the future, they think conditions are going to improve.
\end{abstract}

COVID 19, Emotionality, Pandemic

Citación: MARQUEZ-DE ANDA, Camilo, QUEZADA-FLORES, Ma. De la Luz, SERRANO-TORRES, Ma. Guadalupe y MEJIA-GUERRERO, Mauricio. Emociones frente a la pandemia del COVID-19 de los alumnos del Área Económico Administrativa de la Universidad Tecnológica de León. Revista Transdisciplinaria de Estudios Migratorios. 2020, 6-19: $1-11$

\footnotetext{
*Correspondencia al Autor (correo electrónico: cmarquez@utleon.edu.mx)

$\dagger$ Investigador contribuyendo como primer Autor.
} 


\section{Introducción}

De manera imprevista y vertiginosa entramos en una nueva realidad. Conocimos la existencia de un virus surgido en China. Mientras se creaban historias sobre su procedencia, poder de devastación y veracidad; nos vimos envueltos en la pandemia causada por él. Ya no importa si es real o no, ya tampoco nos importa si viene de los murciélagos o de otros animales, si fue solamente para terminar con la gente mayor, que de alguna manera se constituye como una carga para la economía de las familias, la sociedad y el país. Hoy estamos constatando que los efectos son desastrosos, no solo en cuanto a la salud física, sino también en cuanto a la salud emocional. La sociedad se vio afectada en su totalidad, al desencadenarse un desastre económico y finalmente una crisis política que ha capturado toda la atención global.

Esta investigación forma parte de otras investigaciones realizadas en gran parte del país, sobre cada uno de los aspectos ya mencionados: salud física, salud mental, efectos sociales, efectos económicos y las estrategias y políticas de gestión de la pandemia. En el presente artículo, se presentan los resultados de la investigación realizada a los alumnos de la Universidad Tecnológica de León, con respecto a las emociones y sentimientos que la pandemia ha causado en esta población estudiantil, así como su percepción acerca de cómo se ha manejado la prevención para los contagios y forma particular, la emocionalidad que toda esta situación ha provocado en ellos.

Se eligió este tema debido a la gran importancia que representan las emociones en el ser humano. Las emociones son esencialmente lo que mueve a la humanidad. Si se analiza el comportamiento humano, nos encontraremos que existen en él, emociones, sentimientos y actitudes. Y si buscamos identificar cada una de ellas, tenemos que las actitudes son las decisiones de comportamiento que toma el individuo en respuesta a sus emociones. En cuanto a los sentimientos, son estados de ánimo causados por una emoción. Por tanto, vemos que la causa de nuestro comportamiento es la emoción. (Fuentes, 2017) Por consiguiente, siendo congruentes con la importancia de la emocionalidad de los individuos, nos dimos a la tarea de conocer este aspecto, en el entorno de la pandemia causada por el COVID 19 y los efectos en los alumnos mencionados.
Al hablar de pandemia, dejamos de considerar diversos factores que trae consigo esta situación. La pandemia no es un elemento aislado, sino que trae consigo una serie de factores que tienen efectos en las personas que viven en ella. Estos factores nos conducen a modificar profundamente nuestros estilos de vida y las rutinas a las que tanto nos aferramos. $\mathrm{Y}$ al modificar nuestra forma de vivir, tenemos que formarnos nuevos hábitos de todo tipo; podemos decir que "ya no somos nosotros"; por lo menos no producimos vivencias que nos identificaban como los que éramos antes. Por eso se habla de una nueva normalidad. La anterior ya no existe los que éramos ayer tampoco. Sin embargo, entrar de golpe a ser alguien diferente al que fuimos, nos representa temores; lo anterior ya lo conocíamos y dominábamos en cierta forma; ahora tenemos que construir nuevas relaciones y diferentes, nuevos grupos sociales $y$ con protocolos nuevos de convivencia.

El estar más tiempo en un lugar diferente tiene también cambios en las relaciones. Antes, estábamos ocho horas o más al día en el trabajo, sin contar el tiempo de traslado. Ahora estamos en casa esas ocho horas, más las horas de traslado, que sumando a las que dedicábamos al descanso y atención de la familia, son 24 horas prácticamente, ¡todo el día!. Por otra parte la economía también se vio afectada severamente y esto causa malestar y temor en todos, la incertidumbre reina sobre todas las cosas.

Todas estas situaciones, inciden de manera definitiva en la emocionalidad de las personas que viven en nuestro entorno y con las que continuamente nos relacionamos. Las relaciones pues, se vuelven diferentes y es muy importante conocer cuál es esa emocionalidad. $\mathrm{Si}$ es positiva, se puede generar un ambiente agradable y funcional, pero si es lo opuesto, el ambiente será negativo y disfuncional.

En este artículo analizamos los resultados de la investigación que se llevó a cabo, sobre el tema: "Emocionalidad en los alumnos de la Universidad Tecnológica de León, ante la contingencia". El análisis de los resultados nos ayudará a conocer la realidad que viven los estudiantes y cómo se transformó su contexto, para estar en condiciones, a partir de este conocimiento, de proponer medidas para impulsar la formación de los futuros profesionistas egresados de esta institución. 


\subsection{Justificación}

La contingencia generada ha provocado diferentes estados de ajuste, ya sea del terreno emocional, económico, de salud etc. Para responder a esta situación de cambio y adaptación a la nueva realidad, es necesario cambiar los detonadores para obtener respuestas diferentes y adecuadas a la nueva normalidad. Esto hace necesaria la adopción de técnicas y tácticas diferentes para obtener resultados adecuados en lo que realizamos de manera profesional.

El acompañamiento que se brinda a los alumnos debe cambiar y adecuarse a los nuevos comportamientos para que el apoyo sea pertinente. Para estar en condiciones de brindar esa labor, es necesario conocer las emociones que condicionan los comportamientos de los alumnos. Es por eso que se realiza el presente trabajo de investigación.

\subsection{Alcance}

En el presente proyecto se plantea para realizar una investigación documental sobre COVID-19 y el papel de la emocionalidad en las crisis. También incluye el diseño y validación de un instrumento de recolección de información, que nos permita obtener datos de la emocionalidad de los alumnos. Posteriormente se lleva a cabo un análisis de la información recabada y comentar los resultados y propuestas de acciones.

\subsection{Objetivos}

Objetivo general: Mejorar la pertinencia en el apoyo a los alumnos de las carreras de Administración y turismo del área Económico Administrativa de la Universidad Tecnológica de León, ante las nuevas condiciones originadas por la contingencia causada por el Covid 19, mediante el estudio de los efectos causados en la emocionalidad de este grupo.

Objetivos metodológicos:

- Objetivo No 1: Realizar una investigación documental, sobre la pandemia causada por el Covid 19.

- Objetivo No 2: Realizar una investigación sobre los efectos de la pandemia en la emocionalidad de las personas y su importancia.
- $\quad$ Objetivo No 3: Diseñar un instrumento de recolección de información, para conocer la emocionalidad de los alumnos de las carreras mencionadas.

- Objetivo No 4: Validar el instrumento de recolección de información y aplicarlo a la población referida.

- Objetivo No 5: Analizar los resultados de la información conseguida y exponer las conclusiones del estudio.

- $\quad$ Objetivo No 6: Diseñar una propuesta para el mejoramiento de la pertinencia en el apoyo a los alumnos de las carreras de Administración y turismo del área Económico Administrativa de la Universidad Tecnológica de León.

\section{Marco Teórico}

Objetivo No 1: Realizar una investigación documental, sobre la pandemia causada por el Covid 19.

En diciembre de 2019 se dio a conocer una enfermedad de las vías respiratorias potencialmente mortal en Wuhan provincia de Hubei, China (COVID 19). Esa enfermedad afectó a toda China, no solo en cuestión de salud, sino en otras muchas esferas de la convivencia humana. Luego se extendió rápidamente por todo el mundo con miles de casos confirmados y muertes, transformándose en pandemia y desafiando los sistemas de salud pública.(poudel et al. 2020; citado por Alfonso Urzúa et al.) La velocidad del contagio es uno de los principales problemas, ya que los sistemas de salud puedan colapsar. Además de lo anterior se afecta a personas que tienen otros trastornos de salud, llamados no contagiosos, pero que los vuelve vulnerables ante este nuevo virus, así como también a las personas mayores en las que la enfermedad puede transformarse en grave y su atención urgente. (Guo, Cao, Hong et al. 2020; citado por Alfonso Urzúa et al.)

Existen antecedentes de enfermedades similares. Una de ellas es el síndrome Respiratorio del medio Oriente (MERS) y otra, el Síndrome Respiratorio agudo severo (SARS) de las que podemos extraer evidencia importante para entender al Covid 19. (Alfonso Urzúa et al., 2020) 
Aunado a lo anterior, las enfermedades crónicas no transmisibles (ECNT) son un reto para los Sistemas de Salud a nivel mundial. De acuerdo a las estadísticas de los sistemas de Salud de los diferentes países, esta situación es alarmante porque estas enfermedades se incrementan por el envejecimiento y los hábitos de cada uno de los países. (Valdés, 2020)

Según estadísticas de la OMS, más de 40 millones de personas mueren anualmente por ECNT y son las principales causas de fallecimiento, las enfermedades cardiovasculares (17,7 millones cada año), seguidas del cáncer (8,8 millones), las enfermedades respiratorias (3,9 millones) y la diabetes (1,6 millones). Estos cuatro grupos de padecimientos son responsables de más del $80 \%$ de todas las muertes prematuras por ECNT. (Pérez, 2020)

La OMS alerta sobre el incremento aún mayor para la presente década que comienza. Lo anterior obedece a varias causas: Como ya se mencionó anteriormente, las personas mayores representan una gran problemática. El número de casos afectados cada vez es mayor con sus tasas de incidencia crecientes. Su gradual contribución a la mortalidad general. Además, son la causa más frecuente de discapacidad. También por el costo elevado de la hospitalización, el de su tratamiento médico y el de su posterior rehabilitación. Su emergencia como problema de salud pública, sigue siendo el resultado de diversas modificaciones: cambios sociales, culturales, políticos y económicos que modificaron el estilo y condiciones de vida de un gran porcentaje de la población a escala universal: los determinantes sociales de la salud. (Serra \& Viera, 2020)

Este fenómeno se convirtió muy pronto en pandemia al crecer el número de casos registrados cada día. Igualmente la mortalidad se multiplica y sobre todo en adultos mayores de 60 años y con ECNT en los que presentan complicaciones atípicas, como: febrícula, adinamia, marcada astenia, síntomas confesionales sin síntomas respiratorios plenos y mayor deterioro cognitivo en pacientes con demencia. (Nieto, 2020)

Objetivo No 2: Realizar una investigación sobre los efectos de la pandemia en la emocionalidad de las personas y su importancia
La psicología es la experta en el estudio de emocionalidad por lo que se han revisado algunos artículos sobre estudios psicológicos de las pandemias y concretamente sobre COVID19. Ursúa cometa que existen comportamientos que pueden evitar el contagio y la propagación y discute sobre la posibilidad de que gran parte del problema se pueda evitar cambiando los comportamientos de las personas. (Alfonso Urzúa et al., 2020) afirma que la variable que hace que la enfermedad pueda cerrar fronteras y colapsar sanitaria, política y económicamente a todo el planeta, es el comportamiento humano.

Bayes y Ribes, 1992, argumentaban que la adquisición, proceso y tratamiento es desde un inicio y final, un proceso psicológico. En el caso de la pandemia por COVID-19 la evidencia ha mostrado que todas las medidas preventivas sugeridas incorporan medidas comportamentales. En cuanto a este virus, ya se reportan algunas reacciones observadas en la población, (Torales, O Higgins, CastaldelliMaja \& Ventriglio, 2020). Shingemura, Ursano, Morganstein, Kurosawa\& Benedek (2020) informan que las respuestas emocionales incluyen miedo, e incertidumbre extremos.Citado por Urzúa (Alfonso Urzúa et al., 2020)

Por otra parte, Lozada et al. Comenta que el impacto en la población Española de la información sobre el COVID -19 resaltó la importancia de las emociones en especial las negativas y el aprecio por el apoyo emocional y la empatía. Las investigaciones de los últimos años señalan que las crisis son en realidad lo que la gente piensa sobre ellas y no tanto los hechos en sí. Así Coombs 2007, afirma que "la crisis puede verse como una percepción de un hecho que amenaza a una sociedad y hasta el propio desempeño de las organizaciones. De igual manera, las crisis son en gran parte, perceptivas". También, Kleinnijenhuis et al 2015 asegura que "se puede afirmar que, aunque la crisis tiene un origen real, al final se construye a partir de la interacción entre varios actores, cuyas percepciones producen consecuencias reales" y Utz et al. 2013, nos comentan que "en esta era de comunicación socialmente mediada, los públicos determinan la relevancia, el interés, y la credibilidad de la información recibida". 
Existen componentes racionales y emocionales en a información y de hecho los segundos son determinantes en la construcción mental. Slovic y Burns 2012 afirman que "durante las crisis los receptores no solo confían en lo que piensan de un tema sino también en lo que sienten sobre ese tema". Dunlop et al. 2008 Los individuos necesitan asociar algún tipo de etiqueta emocional a un problema para sentir riesgo por ello. Según Pang, Jin y Cameron, 2009 "en una crisis a medida que el conflicto entre el público y la organización se intensifica las emociones son uno de los anclajes que utilizan los públicos para interpretar lo que está pasando". La carga emocional en una crisis como recuerdan Van der Meer et al. 2014 está presente en un conjunto de personas, no solo en los directamente afectados. (J.C.Lozada díaz, 2020)

En diversas investigaciones encontramos afirmaciones que nos hablan de la importancia de la emocionalidad y por supuesto esta emocionalidad juega un papel importante en las crisis. Hyo J. Kim, glen T. Cameron señalan que las emociones importan en crisis: el papel de la ira y la tristeza en la respuesta del público el encuadre de noticias de crisis y la respuesta a las crisis corporativas. (Hyo J. Kim, 2011)

El filósofo Chileno, Humberto Maturana, afirma que "los seres humanos nos movemos por la emotividad: son las emociones las que nos disponen a hacer todo lo que hacemos". (Fuentes, 2017) "No hay acción humana sin una emoción que la funde y la haga posible como acto". "Los seres humanos somos emocionales, aunque se pretenda renegar de ello. La racionalidad surge de la emocionalidad, de las preferencias y deseos de las personas." (Garrido, 2017)

Dada toda la evidencia que existe sobre la importancia de las emociones en todos los comportamientos humanos y aunado a esto las características que tiene el comportamiento movido por la emoción en la crisis del COVID19 es que se realiza la presente investigación.

Objetivo No 3: Diseñar un instrumento de recolección de información, para conocer la emocionalidad de los alumnos de las carreras mencionadas.
Objetivo No 4: Validar el instrumento de recolección de información y aplicarlo a la población referida.

Con el fin de llevar a cabo este proyecto, se definieron el tipo de investigación, optando por una investigación cualitativa con diferentes variables a investigar. Entre ellas la parte emocional que es la que nos ocupa en este documento. El instrumento de recolección de información, fue un cuestionario que contienen 64 items. Este cuestionario se aplicó de manera virtual a los alumnos de la carrera de Administración, del área Económico Administrativa de la Universidad Tecnológica de León. En total fueron 249 alumnos los que respondieron de manera adecuada el cuestionario en este proyecto. Respecto a las pruebas, el coeficiente de estabilidad se determinó mediante la correlación $r$ de Pearson. Coeficiente de estabilidad de un 0.90, correlación $\mathrm{r}$ de Pearson que nos dio 0.90 y una confiabilidad del 0.80 mediante el alfa de Cronbach.

En este reporte, se seleccionaron solamente los factores que se refieren a las emociones y sentimientos que origina esta pandemia, ya sea por el temor a ser infectados, ellos o sus familias, o por la determinación de estar recluidos en casa, demasiado tiempo, comparado con la situación que vivían anterior a la crisis mencionada.

De toda esta serie de ítems que se refieren a las emociones y sentimientos, se tomaron solamente algunos, que se consideraron más significativos por los resultados encontrados y que reflejan de manera clara, cuál es la emocionalidad que manifiestan los alumnos, respecto al peligro de infección y en cuanto a los cambios que representan, el adquirir nuevos hábitos para la convivencia con sus amigos, sus compañeros de universidad, círculos sociales, culturales y deportivos, con los que tenían relaciones significativas y que por la reclusión existente, no se han podido continuar de la misma manera, o definitivamente no se continuaron.

También se tomaron en cuenta los resultados de la emocionalidad que viven, al estar conviviendo muchas más horas en su casa y lo que esto representa. 
El cambio que se genera al estar cumpliendo con sus actividades escolares, tanto ellos como las demás personas que viven en la misma casa. El tiempo que conviven se ve significativamente incrementado y se generan diferentes relaciones que pueden ser positivas o negativas. Esto genera emocionalidades que serán analizadas en esta sección.

\section{Metodología}

La pandemia por COVID 19 existente, ha provocado una crisis multifactorial. Los efectos de la crisis se han ido considerando y midiendo en cuanto al impacto social, económico, de salud y político; sin embargo, existen efectos secundarios que no se han medido y que afectan de manera diferente al interior de cada país.

Atendiendo a esta necesidad, el cuerpo académico Económico Administrativo de la Universidad Tecnológica de León, emprendió una investigación sobre estos efectos secundarios, en los alumnos de las carreras de Administración y Turismo del área Económico administrativa de dicha universidad, con el objeto de conocer el impacto causado en esta población.

\section{Resultados}

Presentamos a continuación el análisis de los resultados de la investigación, considerando la etapa en la que se encuentran estos jóvenes. Durante estos años que son de preparación para su vida profesional, se encuentran altamente receptivos a la influencia de las condiciones de su entorno. Cuando estas condiciones son totalmente nuevas e inesperadas, podemos apreciar su carácter y capacidad de respuesta para su adaptación a la nueva normalidad que les espera.

En la tabla \# 1 apreciamos que solo una quinta parte, representa las opiniones de los hombres, mientras las otras 4 quintas partes, la de las mujeres. Esto significa que en esta carrera la proporción de mujeres es abrumadoramente mayor que la de los hombres, que generalmente manifiestan preferencia por otras carreras de carácter tecnológico.

Podemos esperar entonces mayor porcentaje de influencia emocional, si nos atenemos a las características del género femenino, que tienden a expresar más fácilmente sus emociones.

\begin{tabular}{|l|r|r|}
\hline Descripción & Número & Porcentaje \\
\hline Hombre & 51 & $20 \%$ \\
\hline Mujer & 198 & $80 \%$ \\
\hline
\end{tabular}

Tabla 1 Genero

Fuente: Elaboración Propia

En la siguiente tabla, apreciamos que la gran mayoría viven con sus padres (76\%) y el 18 $\%$ con su madre. La mayoría son solteros y dependientes de sus dos padres o uno de ellos. Esta palabra "dependientes", nos hace pensar que tienen quién se preocupe por ellos $\mathrm{y}$, en cierta manera, ellos no tienen preocupaciones por otros, además de ellos mismos.

$\%$ y en apoyo de actividades e información un $20 \%$.

\begin{tabular}{|l|r|r|}
\hline \multicolumn{1}{|c|}{ Descripción } & Número & Porcentaje \\
\hline Ambos padres & 188 & $76 \%$ \\
\hline Con alguien más & 6 & $2 \%$ \\
\hline Con mi madre & 46 & $18 \%$ \\
\hline Con mi padre & 7 & $3 \%$ \\
\hline Solo & 2 & $1 \%$ \\
\hline
\end{tabular}

Tabla 2 Actualmente vivo con

Fuente: Elaboración Propia

La siguiente tabla nos muestra la cantidad de personas que habitan en la vivienda de los encuestados. Esta información es importante para determinar con cuántas personas interactúan, cuando están en su casa los encuestados, dado que ahora por la cantidad de tiempo que están recluidos en bastante significativa. Además, existe mayor posibilidad de conflictos, al tener mayor número $\mathrm{y}$ frecuencia de interacciones. Los resultados nos muestran que casi el $80 \%$ conviven con un rango de una a cinco personas; lo anterior nos muestra que son pocas las personas y menores las posibilidades y la oportunidad de surgimiento de conflictos.

\begin{tabular}{|l|r|r|}
\hline Descripción & Número & Porcentaje \\
\hline De 1 a 3 & 31 & $12 \%$ \\
\hline De 4 a 6 & 165 & $66 \%$ \\
\hline De 7 a 9 & 38 & $15 \%$ \\
\hline Más de 10 & 8 & $3 \%$ \\
\hline
\end{tabular}

Tabla 3 ¿Cuántas personas viven en casa incluyéndote? Fuente: Elaboración Propia

De acuerdo a la apreciación sobre la gravedad de la enfermedad que causa la pandemia, casi el $80 \%$ considera que es de grave a muy grave. Esto nos indica que existe la conciencia de que es una situación de cuidado y deben estar alertas para evitar el contagio.

MARQUEZ-DE ANDA, Camilo, QUEZADA-FLORES, Ma. De la Luz, SERRANO-TORRES, Ma. Guadalupe y MEJIA-GUERRERO, Mauricio. Emociones frente a la pandemia del COVID-19 de los alumnos del Área Económico Administrativa de la Universidad Tecnológica de León. Revista Transdisciplinaria de Estudios Migratorios. 2020 


\begin{tabular}{|l|r|r|}
\hline Descripción & Número & Porcentaje \\
\hline Muy grave & 72 & $29 \%$ \\
\hline Grave & 121 & $49 \%$ \\
\hline Regular & 46 & $18 \%$ \\
\hline Poco grave & 10 & $4 \%$ \\
\hline
\end{tabular}

Tabla 4 ¿Qué tan grave consideras que es la infección por coronavirus?

Fuente: Elaboración Propia

En cuanto a la ansiedad que provoca la existencia de esta enfermedad, la mitad considera que se sienten ansiosos. Una tercera parte se siente regularmente ansioso. Solo un $25 \%$ considera que poco o nada.

\begin{tabular}{|l|r|r|}
\hline Descripción & \multicolumn{1}{r|}{ Número } & Porcentaje \\
\hline Muchísimo & 69 & $28 \%$ \\
\hline Mucho & 53 & $21 \%$ \\
\hline Regular & 66 & $27 \%$ \\
\hline Poco & 32 & $13 \%$ \\
\hline Nada & 29 & $12 \%$ \\
\hline
\end{tabular}

Tabla 5 La epidemia del COVID-19, qué tanto te ha hecho sentir Ansioso

Fuente: Elaboración Propia

En cuanto a la preocupación que sienten por la enfermedad, la mitad comentan estar de preocupados a muy preocupados. La tercera parte declara estar regularmente preocupados y el $18 \%$ poco o nada.

\begin{tabular}{|l|r|r|}
\hline Descripción & Número & Porcentaje \\
\hline Muchísimo & 56 & $22 \%$ \\
\hline Mucho & 71 & $29 \%$ \\
\hline Nada & 14 & $6 \%$ \\
\hline Poco & 29 & $12 \%$ \\
\hline Regular & 79 & $32 \%$ \\
\hline
\end{tabular}

Tabla 6 La epidemia del COVID-19, qué tanto te ha hecho sentir Preocupado

Fuente: Elaboración Propia

En cuanto al temor que sienten respecto a la enfermedad, el $30 \%$ dicen que mucho, otro $30 \%$ regular y el $40 \%$ poco o nada. Por alguna razón, la emoción relacionada con el temor la aceptan más de la mitad, pero es menor a otras emociones que se analizaron. Esto puede atribuirse a la cultura existente.

\begin{tabular}{|l|r|r|}
\hline Descripción & Número & Porcentaje \\
\hline Muchísimo & 23 & $9 \%$ \\
\hline Mucho & 45 & $18 \%$ \\
\hline Regular & 75 & $30 \%$ \\
\hline Poco & 61 & $24 \%$ \\
\hline Nada & 45 & $18 \%$ \\
\hline
\end{tabular}

Tabla 7 La epidemia del COVID-19, qué tanto te ha hecho sentir Temeroso

Fuente: Elaboración Propia
En cuanto a la acción de estar alerta, el $42 \%$ comentan que están muy alerta, el 35\% regular y el $23 \%$ poco o nada. Lo que nos da nos da como resultado, que tres cuartas partes están alerta desde regularmente hasta muchísimo, y solo una cuarta parte poco o nada.

\begin{tabular}{|l|r|r|}
\hline Descripción & Número & Porcentaje \\
\hline Muchísimo & 46 & $18 \%$ \\
\hline Mucho & 60 & $24 \%$ \\
\hline Regular & 86 & $35 \%$ \\
\hline Poco & 34 & $14 \%$ \\
\hline Nada & 23 & $9 \%$ \\
\hline
\end{tabular}

Tabla 8 La epidemia del COVID-19, qué tanto te ha hecho sentir Alerta

Fuente: Elaboración Propia

Con relación al sentimiento de aislamiento, casi en $60 \%$ comentan mucho $20 \%$ regular y $20 \%$ de poco a nada. Los resultados nos muestran que este sentimiento es muy fuerte en la mayoría de los encuestados.

\begin{tabular}{|l|r|r|}
\hline Descripción & Número & Porcentaje \\
\hline Muchísimo & 85 & $34 \%$ \\
\hline Mucho & 59 & $24 \%$ \\
\hline Regular & 53 & $21 \%$ \\
\hline Poco & 27 & $11 \%$ \\
\hline Nada & 25 & $10 \%$ \\
\hline
\end{tabular}

Tabla 9 La epidemia del COVID-19, qué tanto te ha hecho sentir Aislado

Fuente: Elaboración Propia

Respecto a la discriminación, comentan 83\% no sentirse así. El 13\% sólo regular.

\begin{tabular}{|l|r|r|}
\hline Descripción & Número & Porcentaje \\
\hline Muchísimo & 4 & $2 \%$ \\
\hline Mucho & 6 & $2 \%$ \\
\hline Regular & 33 & $13 \%$ \\
\hline Poco & 45 & $18 \%$ \\
\hline Nada & 161 & $65 \%$ \\
\hline
\end{tabular}

Tabla 10 La epidemia del COVID-19, qué tanto te ha hecho sentir Discriminado

Fuente: Elaboración Propia

En cuanto a esta variable, el $73 \%$ comentó que mucho y el $15 \%$ regular, lo que nos da casi el $90 \%$ de personas aburridas. 


\begin{tabular}{|l|r|r|}
\hline Descripción & Número & Porcentaje \\
\hline Muchísimo & 149 & $60 \%$ \\
\hline Mucho & 33 & $13 \%$ \\
\hline Regular & 37 & $15 \%$ \\
\hline Poco & 19 & $8 \%$ \\
\hline Nada & 11 & $4 \%$ \\
\hline
\end{tabular}

Tabla 11 La epidemia del COVID-19, qué tanto te ha hecho sentir Aburrido

Fuente: Elaboración Propia

En referencia a sentirse tenso o nervioso, el $40 \%$ manifiesta que una parte del día se sienten así y el $53 \%$ de vez en cuando. Esta situación de sebe al hecho de estar recluidos.

\begin{tabular}{|l|r|r|}
\hline \multicolumn{1}{|c|}{ Descripción } & Número & Porcentaje \\
\hline Casi todo el día & 36 & $14 \%$ \\
\hline Gran parte del día & 64 & $26 \%$ \\
\hline De vez en cuando & 133 & $53 \%$ \\
\hline Nunca & 16 & $6 \%$ \\
\hline
\end{tabular}

Tabla 12 A partir de la situación de "Quédate en casa", me siento tenso (a) o nervioso (a):

Fuente: Elaboración Propia

Por el aislamiento, la mitad manifiesta que ya no disfruta como antes, o que disfruta poco $35 \%$

\begin{tabular}{|l|r|r|}
\hline \multicolumn{1}{|c|}{ Descripción } & Número & Porcentaje \\
\hline Ciertamente, igual que antes & 14 & $6 \%$ \\
\hline No tanto como antes & 118 & $47 \%$ \\
\hline Solamente un poco & 88 & $35 \%$ \\
\hline Ya no disfruto nada & 29 & $12 \%$ \\
\hline
\end{tabular}

Tabla 13 A partir de la situación de "Quédate en casa", sigo disfrutando de las cosas como siempre:

Fuente: Elaboración Propia

En cuanto al temor de que algo fuera a ocurrir, la mitad dice sentirlo y $35 \%$ no siente nada de eso. Otra vez recordemos nuestra cultura respecto al miedo o al temor.

\begin{tabular}{|l|r|r|}
\hline \multicolumn{1}{|c|}{ Descripción } & Número & Porcentaje \\
\hline Sí, y muy intenso & 19 & $8 \%$ \\
\hline Sí, pero no muy intenso & 100 & $40 \%$ \\
\hline Sí, pero no me preocupa & 44 & $18 \%$ \\
\hline No siento nada de eso & 86 & $35 \%$ \\
\hline
\end{tabular}

Tabla 14 Siento una especie de temor como si algo malo fuera a suceder:

Fuente: Elaboración Propia

Respecto de la capacidad de reír el 57\% manifiestan que menos o mucho menos. El 34\% dicen que igual que siempre. Dos terceras parte ríen menos y esto afecta de alguna manera su salud.

\begin{tabular}{|l|r|r|}
\hline \multicolumn{1}{|c|}{ Descripción } & Número & Porcentaje \\
\hline Igual que siempre & 84 & $34 \%$ \\
\hline Actualmente, algo menos & 95 & $38 \%$ \\
\hline Actualmente, en absoluto & 22 & $9 \%$ \\
\hline Actualmente, mucho menos & 48 & $19 \%$ \\
\hline
\end{tabular}

Tabla 15 Soy capaz de reírme y ver el lado gracioso de las cosas:

Fuente: Elaboración Propia

El sentirse saturado de preocupaciones, está presenta en todo el día o gran parte del $42 \%$. $\mathrm{Y}$ del $50 \%$ de vez en cuando. Es difícil que se concentren bien en este estado.

\begin{tabular}{|l|r|r|}
\hline \multicolumn{1}{|c|}{ Descripción } & Número & Porcentaje \\
\hline Gran parte del día & 65 & $26 \%$ \\
\hline Casi todo el día & 40 & $16 \%$ \\
\hline De vez en cuando & 121 & $49 \%$ \\
\hline Nunca & 23 & $9 \%$ \\
\hline
\end{tabular}

Tabla 16 A partir de la situación de "Quédate en casa", tengo la cabeza llena de preocupaciones:

Fuente: Elaboración Propia

En relación al cuidado de su persona, el $39 \%$ comenta que lo sigue haciendo como antes, el $48 \%$ no como antes y un $13 \%$ perdió el interés por él. No olvidemos que la mayoría son mujeres, ellas cuidan mucho este aspecto.

\begin{tabular}{|l|r|r|}
\multicolumn{1}{|c|}{ Descripción } & Número & Porcentaje \\
\hline Me cuido como siempre & 96 & $39 \%$ \\
\hline No me cuido como antes & 52 & $21 \%$ \\
\hline $\begin{array}{l}\text { Es posible que no me cuide } \\
\text { como debiera }\end{array}$ & 67 & $27 \%$ \\
\hline Completamente & 34 & $14 \%$ \\
\hline
\end{tabular}

Tabla 17 Por el distanciamiento social, he perdido el interés por mi aspecto personal:

Fuente: Elaboración Propia

En cuanto a las ilusiones, el $40 \%$ comentan que esperan las cosas con la misma ilusión, el otro $40 \%$ algo menos o mucho menos que antes. Esa emoción positiva disminuye, aunque no de manera definitiva.

\begin{tabular}{|l|r|r|}
\hline Descripción & Número & Porcentaje \\
\hline Como siempre & 114 & $46 \%$ \\
\hline Algo menos que antes & 79 & $32 \%$ \\
\hline Mucho menos que antes & 30 & $12 \%$ \\
\hline En absoluto & 26 & $10 \%$ \\
\hline
\end{tabular}

Tabla 18 A causa del encierro en casa, espero las cosas con ilusión:

Fuente: Elaboración Propia 
En cuanto a la salud mental, opinan que de mucho a muchísimo (33\%), les afecta el encierro, $30 \%$ regular, y la otra tercera parte poco o nada. Esto nos confirma que emocionalmente no están bien.

\begin{tabular}{|l|r|r|}
\hline Descripción & Número & Porcentaje \\
\hline Muchísimo & 36 & $14 \%$ \\
\hline Mucho & 47 & $19 \%$ \\
\hline Regular & 74 & $30 \%$ \\
\hline Poco & 44 & $18 \%$ \\
\hline Nada & 48 & $19 \%$ \\
\hline
\end{tabular}

Tabla 19 ¿A qué grado está teniendo efectos negativos el tener que "quedarse en casa"? En mi salud mental Fuente: Elaboración Propia

En relación a la salud física, una tercera parte de mucho a muchísimo, otra, regular y la última poco o nada. Con dos terceras parte afectados en su salud física, tenemos mucha gente enferma o por lo menos disminuida físicamente.

\begin{tabular}{|l|r|r|}
\hline Descripción & Número & Porcentaje \\
\hline Muchísimo & 34 & $14 \%$ \\
\hline Mucho & 52 & $21 \%$ \\
\hline Regular & 80 & $32 \%$ \\
\hline Poco & 45 & $18 \%$ \\
\hline Nada & 38 & $15 \%$ \\
\hline
\end{tabular}

Tabla 20 ¿A qué grado está teniendo efectos negativos el tener que "quedarse en casa"? En mi salud física Fuente: Elaboración Propia

La frustración por no poder salir expreso la tercera parte que mucho, otra tercera parte regular y la última poco o nada. Por lo que están frustrados con sentimientos agresivos o de desánimo. Emocionalmente negativa.

\begin{tabular}{|l|r|r|}
\hline Descripción & Número & Porcentaje \\
\hline Demasiado & 35 & $14 \%$ \\
\hline Mucho & 45 & $18 \%$ \\
\hline Regular & 76 & $31 \%$ \\
\hline Poco & 57 & $23 \%$ \\
\hline Nada & 36 & $14 \%$ \\
\hline
\end{tabular}

Tabla 21 Durante esta etapa de pandemia Siento frustración por estar en casa y no poder salir Fuente: Elaboración Propia

El $56 \%$ comenta que tiene de regular a mucho miedo. Ese término para la cultura nacional en muy relativo.

\begin{tabular}{|l|r|r|}
\hline Descripción & Número & Porcentaje \\
\hline Demasiado & 19 & $8 \%$ \\
\hline Mucho & 42 & $17 \%$ \\
\hline Regular & 76 & $31 \%$ \\
\hline Poco & 64 & $26 \%$ \\
\hline Nada & 48 & $19 \%$ \\
\hline
\end{tabular}

Tabla 22 Durante esta etapa de pandemia Siento miedo por la situación que se vive

Fuente: Elaboración Propia

Considerando la ansiedad, más de la mitad esta de regular, a demasiado ansiosa. Y no permite tener la calma para trabajar en sus quehaceres normales de la escuela.

\begin{tabular}{|l|r|r|}
\hline Descripción & Número & Porcentaje \\
\hline Demasiado & 32 & $13 \%$ \\
\hline Mucho & 47 & $19 \%$ \\
\hline Regular & 65 & $26 \%$ \\
\hline Poco & 56 & $22 \%$ \\
\hline Nada & 49 & $20 \%$ \\
\hline
\end{tabular}

Tabla 23 Durante esta etapa de pandemia Siento ansiedad por estar en aislamiento

Fuente: Elaboración Propia

En relación al sentimiento de impotencia, el $65 \%$ la manifiestan de regular a demasiado. Es una emoción negativa causada por la pandemia, que perturba su estabilidad emocional

\begin{tabular}{|l|r|r|}
\hline Descripción & Número & Porcentaje \\
\hline Demasiado & 41 & $16 \%$ \\
\hline Mucho & 47 & $19 \%$ \\
\hline Regular & 69 & $28 \%$ \\
\hline Poco & 52 & $21 \%$ \\
\hline Nada & 40 & $16 \%$ \\
\hline
\end{tabular}

Tabla 24 Durante esta etapa de pandemia Siento impotencia de no poder hacer lo que me gusta fuera de casa Fuente: Elaboración Propia

A pesar de la emocionalidad que tienen los alumnos, no se manifiestan indiferentes ante esta crisis. El 90\% declara no sentirse indiferente.

\begin{tabular}{|l|r|r|}
\hline Descripción & Número & Porcentaje \\
\hline Demasiado & 15 & $6 \%$ \\
\hline Mucho & 14 & $6 \%$ \\
\hline Regular & 72 & $29 \%$ \\
\hline Poco & 78 & $31 \%$ \\
\hline Nada & 70 & $28 \%$ \\
\hline
\end{tabular}

Tabla 25 Durante esta etapa de pandemia Siento indiferencia por esta situación Fuente: Elaboración Propia 
En esta pandemia, más del $80 \%$ se sientes felices de convivir con sus seres queridos, y solo el $20 \%$ poco o nada. Esto nos habla de familias bien integradas.

\begin{tabular}{|l|r|r|}
\hline Descripción & Número & Porcentaje \\
\hline Demasiado & 68 & $27 \%$ \\
\hline Mucho & 60 & $24 \%$ \\
\hline Regular & 75 & $30 \%$ \\
\hline Poco & 27 & $11 \%$ \\
\hline Nada & 19 & $8 \%$ \\
\hline
\end{tabular}

Tabla 26 Durante esta etapa de pandemia Siento alegría por convivir con mis seres queridos

Fuente: Elaboración Propia

El $80 \%$ manifiesta la seguridad que les proporciona el estar en familia durante esta crisis.

\begin{tabular}{|l|r|r|}
\hline Descripción & Número & Porcentaje \\
\hline Demasiado & 58 & $23 \%$ \\
\hline Mucho & 66 & $27 \%$ \\
\hline Regular & 71 & $29 \%$ \\
\hline Poco & 36 & $14 \%$ \\
\hline Nada & 18 & $7 \%$ \\
\hline
\end{tabular}

Tabla 27 Durante esta etapa de pandemia Siento calma y seguridad de estar en mi casa o en un lugar seguro Fuente: Elaboración Propia

Casi el $90 \%$ siente agradecimiento por estar a salvo.

\begin{tabular}{|l|r|r|}
\hline Descripción & Número & Porcentaje \\
\hline Demasiado & 82 & $33 \%$ \\
\hline Mucho & 67 & $27 \%$ \\
\hline Regular & 66 & $27 \%$ \\
\hline Poco & 16 & $6 \%$ \\
\hline Nada & 18 & $7 \%$ \\
\hline
\end{tabular}

Tabla 28 Durante esta etapa de pandemia Siento agradecimiento de estar a salvo

Fuente: Elaboración Propia

El hecho de estar en su casa les produce al $70 \%$ entusiasmo de regular a demasiado. Les gusta estar en casa por la sensación de seguridad pero les produce otros conflictos.

\begin{tabular}{|l|r|r|}
\hline Descripción & Número & Porcentaje \\
\hline Demasiado & 21 & $8 \%$ \\
\hline Mucho & 49 & $20 \%$ \\
\hline Regular & 102 & $41 \%$ \\
\hline Poco & 51 & $20 \%$ \\
\hline Nada & 26 & $10 \%$ \\
\hline
\end{tabular}

Tabla 29 Durante esta etapa de pandemia Siento entusiasmo al estar en casa

Fuente: Elaboración Propia
El $82 \%$ manifiesta estar optimista del futuro, tienen la esperanza de que todo cambiará para bien.

\begin{tabular}{|l|r|r|}
\hline Descripción & Número & Porcentaje \\
\hline Demasiado & 57 & $23 \%$ \\
\hline Mucho & 60 & $24 \%$ \\
\hline Regular & 87 & $35 \%$ \\
\hline Poco & 33 & $13 \%$ \\
\hline Nada & 12 & $5 \%$ \\
\hline
\end{tabular}

Tabla 30 Durante esta etapa de pandemia Siento optimismo de que todo estará bien

Fuente: Elaboración Propia

\section{Conclusiones}

Las investigaciones realzadas en la Universidad Tecnológica de León, nos proporcionaron diversos resultados que nos permitieron un análisis satisfactorio, sobre los sentimientos y emociones que, a causa de la pandemia y las condiciones de reclusión, experimentan los alumnos. Lo anterior nos arroja una luz sobre el estado emocional en que se encuentran. Con la comprensión de esta realidad, estaremos en condiciones de establecer proyectos, que apoyen a los alumnos para poder asegurar su desarrollo profesional, de una manera más congruente y pertinente.

Por medio de esta investigación, sabemos que nuestros alumnos, conocen que la enfermedad denominada COVID 19, es de gravedad y puede provocar la muerte. Esto naturalmente causa en ellos diferentes emociones como: ansiedad, preocupación y temor. Esta pandemia, llevó a una situación de reclusión de las personas en sus casas para evitar la exposición al contagio y la propagación de este virus. El confinamiento de los alumnos, causó efectos secundarios, ya que este encierro originó un cambio en los hábitos de vida.

Al estar más tiempo en sus casas, se sienten aislados, se aburren al no poder realizar las actividades al aire libre o fuera de sus casas. Esto hace que ya no disfrute como antes de las actividades sociales. Sienten temor por lo que puede ocurrir, dado que están expuestos de manera constante a las noticias sobre los estragos de la pandemia. Ríen menos y se sienten saturados por las preocupaciones. Esto por consiguiente no les permite desarrollar plenamente sus capacidades de aprendizaje. 
El encierro les afecta de manera que la ilusión por las cosas ha disminuido y sienten gran frustración e impotencia ante esta nueva forma de vivir. Su salud física y mental se han visto afectadas lo que les genera miedo. A raíz de todo esto, vemos que, en efecto la situación de la pandemia, y la decisión de confinamiento, sí han afectado tanto económicamente, como a la salud, la sociedad en general, la situación política y la manera en que la gente se desempeñaba en su vida daría. Pero también ha traído afectaciones secundarias, como las que mencionamos en las emociones y los sentimientos de todas las personas. Cabe mencionar que, a pesar de todo, manifiestan la esperanza de que el futuro sea mejor para todos.

\section{Recomendaciones}

La primera recomendación que se genera es el cuidado en la información que se maneja en torno a la pandemia. Esta información debe ser veraz clara y oportuna, de manera que no genere falsas expectativas. La segunda recomendación es el cambio de comportamientos en relación a las condiciones de higiene personal, doméstica y laboral.

Generar conciencia de que la emocionalidad de los alumnos no solo obedece a la pandemia, sino también y de manera impactante, a las condiciones sociales $y$ económicas de sus familias.

Diseñar proyectos que puedan ser consultados de manera virtual y a diferentes tiempos para que estén los documentos disponibles y puedan tener acceso en el momento que puedan realizarlo. Habrá muchas cosas más que se puedan implementar y con el tiempo y en base a esta investigación e irán implementando.

\section{Referencias}

Alfonso Urzúa et al. (30 de Marzo de 2020). La psicología en la prevención y manejo del COVID-19 Aportes desde la evidencia. Obtenido de Psicología y pandemia : psicologíaypandemia.pdf(unam.mx)

Fuentes, F. (24 de Marzo de 2017). La objetividad según Maturana. Obtenido de La objetividad según Maturana: https://www.laobjetividad-segun-

maturana/ffuente.es
Garrido, M. (2017). Transformación a convivencia. Santiago de Chile: Dolmen Ediciones.

Hyo J. Kim, G. T. (10 de Enero de 2011). Emotions Matter in Crisis: The Role of Anger and Sadness in the Publics". Obtenido de Emotions Matter in Crisis: The Role of Anger and Sadness in the Publics": https://www. Framingandcorpotated/

J.C.Lozada díaz, R. F. (30 de Marzo de 2020). Comunicación gubernamental y emociones en la crisis del COVID-19 en España. Obtenido de Comunicación gubernamental y emociones en la crisis del COVID-19 en España.: https://www.doi.org/10.4185/RICS-2020-1467

Nieto, C. (23 de Marzo de 2020). Mayor vulnerabilidad y sintomatología atípica en pacientes mayores con COVID-19. Obtenido de Mayor vulnerabilidad y sintomatología atípica en pacientes mayores con COVID-19: https://wwwepaanol.medscape.com/verarticulo/ 5905380

Pérez, T. (21 de Enero de 2020). Retos de la salud urgentes para la próxima década según la OMS. Obtenido de Retos de la salud urgentes para la próxima década según la OMS: https://terezaperez.net/2020/01/21/retos-desalud-urgentes-para-la-proxima-decada-segunla-oms/

Serra, M., \& Viera, M. (23 de Abril de 2020). Las enfermedades crónicas no tasmisibles. Magnitud actual y tendencias futuras. Obtenido de Las enfermedades crónicas no tasmisibles. Magnitud actual $y$ tendencias futuras: https://revfinlay.sld.cu/index.php/finlay/article/ view/561.

Valdés, M. Á. (30 de Junio de 2020). Las enfermedades crónicas notrasmisibles y la pandemia por COVID-19. Obtenido de Las enfermedades crónicas notrasmisibles y la pandemia por COVID-19: scielo,sld.cu/scielo.php?d=s2221-

24342020000200078\&sript=sci:arttext\&tlng=pt 